Z Rheumatol $2010 \cdot 69: 295-297$

DOI 10.1007/s00393-009-0521-8

Online publiziert: 20. Mai 2010

(c) Springer-Verlag 2010

G. Burmester ${ }^{1} \cdot$ M. Schneider ${ }^{2} \cdot$ E. Genth ${ }^{3}$

${ }^{1}$ Klinik für Rheumatologie, Charité-Universitätsmedizin Berlin, Campus Mitte, Berlin

${ }^{2}$ Klinik für Endokrinologie, Diabetologie und Rheumatologie,

Heinrich-Heine-Universität Düsseldorf

${ }^{3}$ Rheumaklinik und Rheumaforschungsinstitut, Aachen

\title{
Leitlinien - ein langer Weg in die Praxis?
}

management in Marburg, dessen wissenschaftliche Leiterin Frau Prof. Kopp ist. Wesentlich für die Akzeptanz und Wirksamkeit sind ihre hohe methodische und fachliche Qualität. In Deutschland haben die AWMF und das Ärztliche Zentrum für Qualität in der Medizin ( 2005 die Anforderungen an hochwertige Leitlinien im Deutschen Leitlinienbewertungsinstrument (DELBI) zusammengefasst. Die Kernaspekte des aus 34 Kriterien bestehenden DELBI-Instruments sind die Repräsentativität des Leitliniengremiums, die Evidenzbasierung und die strukturierte und dokumentierte Konsensfindung. Dabei orientiert sich die Repräsentativität an der Zielgruppe der Leitlinienempfehlungen und ist wesentlich für Akzeptanz der Leitlinien in der Praxis.

Nach der Qualität werden Leitlinien in 3 Klassen eingeteilt. S1-Leitlinien basieren primär auf Expertenmeinung, S2-Leilinien auf bester verfügbarer Evidenz oder strukturiertem Expertenkonsensus, S3Leitlinien auf beidem. Aktuell liegen bei der AWMF 557 S1-Leitlinien, 125 S2-Leitlinien und $75 \mathrm{~S}_{3}$-Leitlinien vor. Wenn innerhalb von 5 Jahren keine Aktualisierung erfolgt, wird eine Leitlinie aus der AWMFListe im Internet genommen.

In den letzten Jahren wurde eine Vielzahl von Leitlinien in anderen Ländern entwickelt. Des Weiteren haben internationale Gremien und Fachgesellschaften Leitlinien und Empfehlungen entwickelt, die teilweise keine gute Übereinstimmung zeigen. In der Rheumatologie gibt es eine wachsende Zahl von Empfehlungen der EULAR („European League Against
Rheumatism“). Über einige dieser Empfehlungen wurde in der Zeitschrift für Rheumatologie berichtet.

Die Methodik der Erstellung von Empfehlungen durch die EULAR wird von M. Dougados in diesem Heft beschrieben. Während die Leitlinienentwicklung in Deutschland nur mit deutschen Experten arbeitet, nehmen an den EULAR-Empfehlungen Experten verschiedener Länder und damit auch verschiedener Gesundheits- und unterschiedlicher Ausbildungssysteme teil. Das methodische Fundament der Qualitätssicherung ist das AGREEInstrument, das auch dem DELBIVerfahren zugrunde liegt, das aber für die deutschen Verhältnisse modifiziert wurde. Schon das Auswahlverfahren des Leitliniengremiums weist deutlich Unterschiede zwischen den EULAR-Empfehlungen und den deutschen nationalen Leitlinien auf. Es fehlen noch systematischen Analysen, inwiefern dadurch deutsche von europäischen Empfehlungen abweichen können.

Der Beitrag von Becker et al. in diesem Heft nimmt Bezug auf die Leitlinienaktivitäten der EUSTAR für die Therapie der systemischen Sklerose.

\section{- Die Problematik der internationalen Leitlinien liegt vor allem in der Umsetzung.}

Verschiedene Länder weisen deutliche Unterschiede in den Gesundheitssystemen und anderen lokalen Bedingungen auf. Dies wirkt sich weniger im Bereich der besten verfügbaren Evidenz aus, die meist 
eine globale Gültigkeit hat, wohl aber im Bereich der Handlungsempfehlungen, die bezogen auf die regionalen Bedingungen konsentiert werden müssen. Die Leitlinienkommission der AWMF hat diese Entwicklung in ihren Qualitätskriterien berücksichtigt, indem sie für die Erstellung einer neuen Leitlinie auch eine systematische Recherche und Bewertung verfügbarer nationaler und internationaler Leitlinien fordert.

Die Deutsche Gesellschaft für Rheumatologie (DGRh) ist als wissenschaftliche Fachgesellschaft in die Entwicklung verschiedener Leitlinien eingebunden. Mitglieder der Kommission Osteologie der DGRh sind in der Arbeitsgruppe des Dachverbands Osteologie (DVO) für die Leitlinie zur Prophylaxe, Diagnostik und Therapie der Osteoporose bei Erwachsenen. Der Beitrag von J. Braun und J. Pfeilschifter zur leitliniengerechten Diagnostik und Therapie der Osteoporose - als CME-Beitrag in diesem Heft - fasst die wichtigen Aspekte dieser Leitlinie zusammen.

Von der DGRh autorisierte Experten haben auch an der Nationalen Versorgungsleitlinie Kreuzschmerz mitgewirkt, die derzeit zur Kommentierung eingesehen werden kann, und sind an der Entwicklung der Leitlinien zum chronischen Rückenschmerz und zum Fibromyalgiesyndrom beteiligt. Die Leitlinienkommission der DGRh berät den Vorstand in Fragen der Leitlinienentwicklung und schließt derzeit die zweite Aktualisierung der Leitlinie „Management der frühen rheumatoiden Arthritis“ ab. Die DGRh orientiert sich in der Leitlinienentwicklung an den Qualitätskriterien der AWMF und des ÄZQ. Diese Qualitätsstandards sind auch für Entwicklung von Empfehlungen in den Kommissionen maßgeblich.

Seit Jahrzehnten arbeitet die Kommission Pharmakotherapie der DGRh daran, unabhängige Stellungnahmen zu pharmazeutischen Produkten für die Rheumatologen $\mathrm{zu}$ formulieren. Diese Emp- fehlungen werden auf Veranlassung des Vorstands und angeregt durch den Beirat der DGRh regelmäßig aktualisiert. Deshalb ist es richtig und wichtig, dass sich die DGRh in der wissenschaftlich und ökonomisch kritischen Frage von Therapieempfehlungen und Leitlinien bei häufigen rheumatischen Erkrankungen in die Pflicht nimmt und solche Empfehlungen regelmäßig veröffentlicht. Die wissenschaftliche Fachgesellschaft hat hier die wichtige Aufgabe und den Anspruch der fachlichen Meinungsführerschaft, dem sie durch Qualitätsorientierung, Aktualität und Neutralität ihrer Empfehlungen gerecht werden muss und den sie durch offene Diskussion und Überzeugung auch in den eigenen Reihen vertreten muss.

\section{$>$ Leitlinien sind Empfehlungen und keine Richtlinien mit Gesetzescharakter}

Über die Implementation von Leitlinien in Deutschland ist wenig bekannt. Es sind Empfehlungen und keine Richtlinien mit Gesetzescharakter. Ihre Effekte sind wenig untersucht. Der Betrag von A. Zink et al. unternimmt den Versuch zu bewerten, wie leitliniengerecht die rheumatologische Versorgung ist, wobei offen bleibt, inwiefern leitlinienkonforme Praxis sich in Abhängigkeit oder unabhängig von der Kenntnis der Leitlinie entwickelt hat. Die Diskussion führt aber zu mehreren wichtigen Punkten der Umsetzung von Leitlinien. Leitlinien beschreiben die Wissensgrundlage und geben konsentierte Handlungsempfehlungen auch in Bezug auf eine frühe Therapie rheumatischer Erkrankungen, sie geben aber nicht vor, wer der Handelnde ist und wie die Aufgabenverteilung in einer Handlungskette ist. Überweisung und kooperative Behandlung ist daher unabhängig vom behandelnden Arzt abhängig von dessen Wissen und Können.

Bei dem großen Anliegen nach Qualität in der Versorgung durch leitlinienbezogenes Handeln wünscht man sich ein klares Bekenntnis dazu auch von Seiten der Kostenträger. Qualität hat ihren Preis! Gerade in der Rheumatologie wird deutlich, dass sie bei einer Betrachtung der Gesamtkosten der Krankheitsfolgen einen deutlichen Nutzen hat.

Ihre

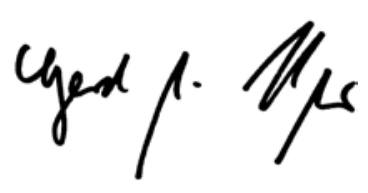

G. Burmester

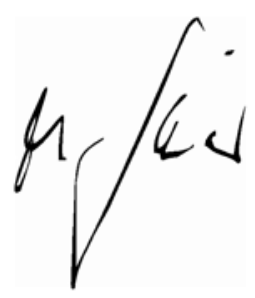

M. Schneider

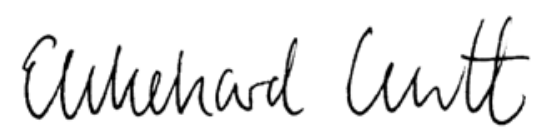

E. Genth

\section{Korrespondenzadresse \\ Prof. Dr. E. Genth}

Rheumaklinik und Rheumaforschungsinstitut

Burscheider Markt 24, 52066 Aachen

mail@ekkehard-genth.de 
Hier steht eine Anzeige.

算 Springer 